\title{
SQLE Promotes Differentiation and Apoptosis of Bovine Skeletal Muscle-Derived Mesenchymal Stem Cells
}

\author{
Ruimen Zhang, ${ }^{1,2, *}$ Yanfei Deng, ${ }^{1,2, *}$ Qiao Lv, ${ }^{1,2}$ Qinghua Xing, ${ }^{1,2}$ Yu Pan,, Jingyuan Liang, ${ }^{1,2}$ \\ Mingsheng Jiang, ${ }^{2}$ Yingming Wei, ${ }^{2}$ Deshun Shi, ${ }^{1,2}$ Bingkun $\mathrm{Xie}^{3},{ }^{3}$ and Sufang Yang ${ }^{1,2}$
}

\begin{abstract}
In this study, Squalene epoxidase ( $S Q L E)$ overexpression vector was transfected into bovine skeletal musclederived mesenchymal stem/stromal cells (MSCs) to study the molecular mechanism of SQLE regulating meat quality through myogenesis. We initially profiled the expression of $S Q L E$ in cattle embryos and adults, in the muscle tissue of four different cattle varieties, and in 11 different tissues/organs of Guangxi cattle variety. Subsequently, we isolated and cultured bovine skeletal muscle-derived MSCs and detected the expression of $S Q L E$ during cell proliferation and differentiation. Then, we constructed a bovine $S Q L E$ overexpression vector and transfected it into bovine skeletal muscle-derived MSCs by liposome transfection. Cell Counting Kit- 8 (CCK-8), 5-ethynyl-20-deoxyuridine (EdU), flow cytometry, immunofluorescence, and quantitative polymerase chain reaction assays were used to characterize cell proliferation and differentiation in detail. The results showed that the relative expression level of bovine $S Q L E$ gene in brain tissue was the highest, and in adult muscle tissue was significantly higher than that in embryonic stage. Especially, the expression of SQLE was significantly upregulated in cell differentiation stage. Furthermore, the proliferation, cell cycle, apoptosis, and myoblast differentiation assays indicated that $S Q L E$ significantly promoted the differentiation and apoptosis of bovine skeletal muscle-derived MSCs, but inhibited their proliferation. In conclusion, our study reveals the role of $S Q L E$ in myoblast differentiation. These results will provide new clues for the regulation network of bovine muscle development.
\end{abstract}

Keywords: bovine, SQLE gene, skeletal muscle-derived MSCs, proliferation, differentiation

\section{Introduction}

A T PRESENT, THE QUANTITY OF BEEF that the market supply cannot meet people's demand, especially the high-quality beef. In the field of feed processing technology and growth hormone and other drug research, scientists have made impressive improvements in meat production and increased meat production (Du and Carlin, 2012; Duarte et al., 2013). In recent years, many candidate genes of $M y o D$, $M y o G$, and MRFs myogenic transcription factors, which are involved in the process of myogenesis and muscle regeneration, have been discovered one after another in the in vitro study of muscle cell growth and development upsurge (Zammit, 2017). Meanwhile, in the field of noncoding RNA, a large number of miRNAs (Mok et al., 2017), lncRNAs (Li et al., 2018c), and emerging circRNAs (Wei et al., 2017) have been found to play an important role in the proliferation and differentiation of bovine muscle cells in vitro, such as mir-7, mir-138, lncRNA (MDNCR/H19) (Li et al., 2018b), circFGFR4 (Li et al., 2018a), and so on.

Based on the previously accumulated molecular mechanisms, we need to explore new myogenic regulators, and use muscle cell lines and emerging biotechnology to study the epigenetic modification of candidate genes in muscle cells. However, knowledge gained from such studies is scarce.

In mammals, Squalene epoxidase (SQLE) is an enzyme that converts squalene to 2,3-oxidosqualene, in the early stage of cholesterol generation (Garcia-Bermudez et al., 2019). In one study, scientists performed genome arrays on bovine muscle tissues with different intramuscular fat content. Twenty-one differentially expressed genes were identified, and the SQLE gene was one of them. However, the

\footnotetext{
${ }^{1}$ State Key Laboratory for Conservation and Utilization of Subtropical Agro-Bioresources, Nanning, Guangxi, China.

${ }^{2}$ College of Animal Science and Technology, Guangxi University, Nanning, Guangxi, China.

${ }^{3}$ Guangxi Key Laboratory of Livestock Genetic Improvement, Guangxi Institute of Animal Sciences, Nanning, Guangxi, China.

*These authors contributed equally to this work.
} 
biological function of $S Q L E$ in muscle development is not yet fully understood. Next, in pig breeding, scientists found that $S Q L E$ was differentially expressed in the dorsal longest muscle tissue of pigs with different genotypes, and revealed that the SNP site of SQLE was associated with backfat thickness, carcass weight, meat color, and other meat quality traits (Ha et al., 2017). At the same time, they preliminarily explored the effect(s) of SQLE gene on the differentiation of muscle cells and adipocytes in vitro using siRNA technology.

The animal body has a remarkable ability to regenerate and heal damaged tissues. This ability is attributed to a special population of multipotent progenitor cells, known as mesenchymal stem/stromal cells (MSCs) (Beane et al., 2014). MSCs can be divided into bone marrow MSCs, umbilical cord MSCs, amniotic membrane MSCs, muscle MSCs (Camernik and Zupan, 2018), etc. MSCs are capable of self-renewal and multidirectional differentiation, and can be differentiated into many cell types under different conditions, including osteoblasts, chondrocytes, nerve cells, adipocytes, myoblasts, etc.

Moreover, they are widely used in the research of obesity, diabetes, muscle development, and other aspects (Nakamura et al., 2015). As we know, muscle is one of the important factors affecting the production performance and meat quality of animals (Hernandez-Hernandez et al., 2017), so it is of great significance to study the proliferation, directional differentiation, and regulatory mechanism of animal muscle MSCs to regulate the mechanism of muscle growth.

In this study, we transfected $S Q L E$ overexpression vector on bovine skeletal muscle-derived MSCs to study the molecular mechanism of SQLE regulating meat quality through myogenesis. Our results will provide new clues to the regulatory network of muscle development in bovine.

\section{Materials and Methods}

\section{Bovine tissue sample preparation}

In this study, all animal samples from Guangxi cattle, buffalo, Chinese Holstein, Limousin at embryonic stage (90 days), and adult stage (30 months old) were collected at a local slaughterhouse in Nanning, Guangxi province. Samples, including muscle, liver, heart, lung, spleen, kidney, brain, and adipose tissues, were collected and immediately frozen in liquid nitrogen. In addition, myoblasts were saved in our laboratory. The samples were kept at $-80^{\circ} \mathrm{C}$ until RNA isolation. Our study was conducted in accordance with the State Key Laboratory for Conservation and Utilization of Subtropical Agro-bioresources Guide for the Care and Use of Laboratory Animals.

\section{RNA isolation, cDNA synthesis, and real-time quantitative polymerase chain reaction}

Total RNA extraction, cDNA synthesis, and real-time quantitative polymerase chain reaction (qPCR) were performed as previously described (Zhu et al., 2018). The expression levels of the transcript mRNAs were calculated by the $2^{-\Delta \Delta \text { ct }}$ method. $\beta$-actin was used as the internal control for normalization of the data, and the cattle lung tissue was considered as a control; others were test samples, and cDNA was prepared by the above methods; PCR and agarose gel were used for analysis. All spanning the distal ends of mRNAs with sequence specificity checked using BLAST, qPCR primers are listed in Supplementary Table S1. For each time point, qPCR was done on three biological replicates.

\section{Gene cloning and vector construction}

The full-length sequence of the ORF (Open Reading Frame) region of SQLE (Supplementary Table $\mathrm{S} 1$ ) was amplified using Q5 ${ }^{\circledR}$ Super fidelity $2 \times$ Master Mix (DNA polymerase, NEW ENGLAND Biolabs ${ }^{\circledR}$ ). In addition, bioinformatics analysis of the ORF region of $S Q L E$ gene was carried out according to the method as previously described (Zhu et al., 2018). To explore the role of $S Q L E$ in bovine MSCs, SQLE overexpression vector was constructed. The genomic region of the $S Q L E$ gene was amplified using Q5 Super fidelity $2 \times$ Master Mix, and PCR primers are listed in Supplementary Table S1. In the end, full-length $S Q L E$ was amplified and cloned into the PMXsIRES-GFP vector (Invitrogen, Carlsbad, CA) to obtain their overexpression plasmid (PMXs-SQLE). All constructs were verified by sequencing.

\section{Cell culture and treatment}

Primary bovine skeletal muscle-derived MSCs were isolated and cultured from bovine longissimus muscle as previously described (Camernik and Zupan, 2018). HEK293T cells and MSCs were cultured in high-glucose Dulbecco's Modified Eagle's Medium (DMEM) with $4.5 \mathrm{~g} / \mathrm{L}$ D-glucose supplemented with fetal bovine serum (Hyclone; $10 \%$ and $20 \%$, respectively) and antibiotics (1\% penicillin and streptomycin; growth medium) at $5 \% \mathrm{CO}_{2}, 37^{\circ} \mathrm{C}$. To induce MSCs' myogenic differentiation, cells were switched to the differentiation medium (DMEM, 2\% horse serum in nearly 90\% confluence. HEK293T cells and MSCs were transfected with PMXs-SQLE (treatment group) and PMXsIRES-GFP (control group) using X-treme GENE HP DNA Transfection Reagent (Roche, Basel, Switzerland) when cell confluence reached $\sim 70 \%$, and the wild-type (WT) cells did not undergo any treatment.

\section{Cell proliferation assay}

To gain insights into the effect of SQLE on MSC's proliferation, the Cell Counting Kit-8 (CCK-8) (Multisciences, Hangzhou, China) and 5-ethynyl-20-deoxyuridine (EdU) incorporation assays (RiboBio, Guangzhou, China) were performed. For the CCK-8 assay, cells were plated into 96well culture plates at a density of $1 \times 10^{4}$ cells/well in $100 \mu \mathrm{L}$ culture medium, and each treatment group had eight independent replicates. After 24 hours of culture at $37^{\circ} \mathrm{C}$, $10 \mu \mathrm{L}$ of CCK-8 reagent was added to each well and incubation was continued for 3-4 hours. The absorbance value of all samples was detected using an automatic microplate reader (Molecular Devices, Sunnyvale) at $450-\mathrm{nm}$ wavelength. The Cell-Light EdU DNA Cell Proliferation Kit was also used to assess the cell proliferation, according to the manufacturer's instructions, and each treatment group had three independent replicates.

Finally, cell samples from the treatment and control groups were collected, respectively, and qPCR was used to detect mRNA expression levels of PCNA, CyclinD1, and $B c l$-2. Primers are listed in Supplementary Table S1. 


\section{Flow cytometry for the cell cycle and apoptosis assays}

We analyzed the cell cycle of different treatment groups using the Cell Cycle Testing Kit (MultiSciences). Bovine skeletal muscle-derived MSCs were grown in six-well plates $\left(1 \times 10^{6}\right.$ cells/well $)$ with $2 \mathrm{~mL}$ culture medium. After treatment for 24 hours, cells were harvested and washed in phosphate-buffered saline (PBS) buffer. Then, $1 \mathrm{~mL}$ of DNA strain solution and $10 \mu \mathrm{L}$ of permeabilization solution were added to the resuspended cells. After incubating for 30 minutes in the dark at room temperature, the cell suspension was used for flow cytometry (FACS Canto II; BD BioSciences), and each treatment group had three independent replicates.

Cell apoptosis was measured by Annexin V-Fluorescein Isothiocyanate/Propidium Iodide (V-FITC/PI) staining assay. After incubation, cells with different treatment were washed three times with PBS buffer and then resuspended in $500 \mu \mathrm{L} 1 \times$ binding buffer. Then, cells were incubated for 10 minutes in the dark at room temperature in the presence of Annexin V-FITC ( $5 \mu \mathrm{L})$ and PI (10 $\mu \mathrm{L}$; Multisciences). Afterward, cells were analyzed using flow cytometry, and each treatment group had three independent replicates. Cells stained with only Annexin $\mathrm{V}$ were evaluated as being in early apoptosis; cells stained with both Annexin V and PI were evaluated as being in late apoptosis stage.

In the end, cell samples from the treatment and control groups were collected, respectively, and qPCR was used to detect mRNA expression levels of BAX, Caspase $9, P 21$, and $P 53$. Primers are listed in Supplementary Table S1.

\section{Immunofluorescence and microscopy}

Immunofluorescence assay was used to study the effects of SQLE on MSC's myogenic differentiation. Primary bovine MSCs at the stage of $95 \%$ confluence were washed three times with PBS buffer ( $\mathrm{pH} 7.4$ ), fixed in PBS containing $4 \%$ paraformaldehyde for 20 minutes, and permeabilized in PBS containing $0.5 \%$ Triton X-100 for 10 minutes. Immunostaining was carried out as follows: cells were in- cubated overnight at $4{ }^{\circ} \mathrm{C}$ with the primary anti-MyoDl (1:200; Abcam, Cambridge, MA), diluted in 5\% bovine serum albumin. Afterward, we washed cells with PBS and incubated them at room temperature for 2 hours with the corresponding secondary antibody goat anti-mouse IgG H\&L (1:1000; Abcam) diluted in 1\% bovine serum albumin in PBS. We visualized DNA using $5 \mathrm{mg} / \mathrm{mL}$ DAPI staining.

Finally, we washed the prepared cells three times with PBS and observed them under a fluorescence microscope (DM5000B; Leica, Germany).

In the end, cell samples from the treatment and control groups, respectively, were collected, and qPCR was used to detect mRNA expression levels of SQLE, MyoD1, MyoG, and $M y H C$. Primers are listed in Supplementary Table S1.

\section{Statistical analysis}

The quantitative results are presented as mean \pm standard error of the mean based on at least three independent experiments. Significant variance by treatments in comparison to the untreated sample was determined by one-way ANOVA performed in GraphPad Prism version 6.0 (GraphPad Software, La Jolla, CA). Differences were considered significant at $p$-values $\leq 0.05$.

\section{Results}

\section{Expression profile of bovine SQLE in various tissue and organ types}

To study the biological function of SQLE gene in muscle development, qPCR was used to detect the expression level of $S Q L E$ in different tissues and organs of cattle, and detected the $S Q L E$ expression level in embryonic stage and adult stage of four varieties of beef cattle expression information of the longissimus muscle tissue.

The results showed that among different tissues and organs, the expression level of SQLE gene in brain tissue was the highest, which was 2.26-fold of the expression in longissimus muscle tissue, and the lowest in lung tissue
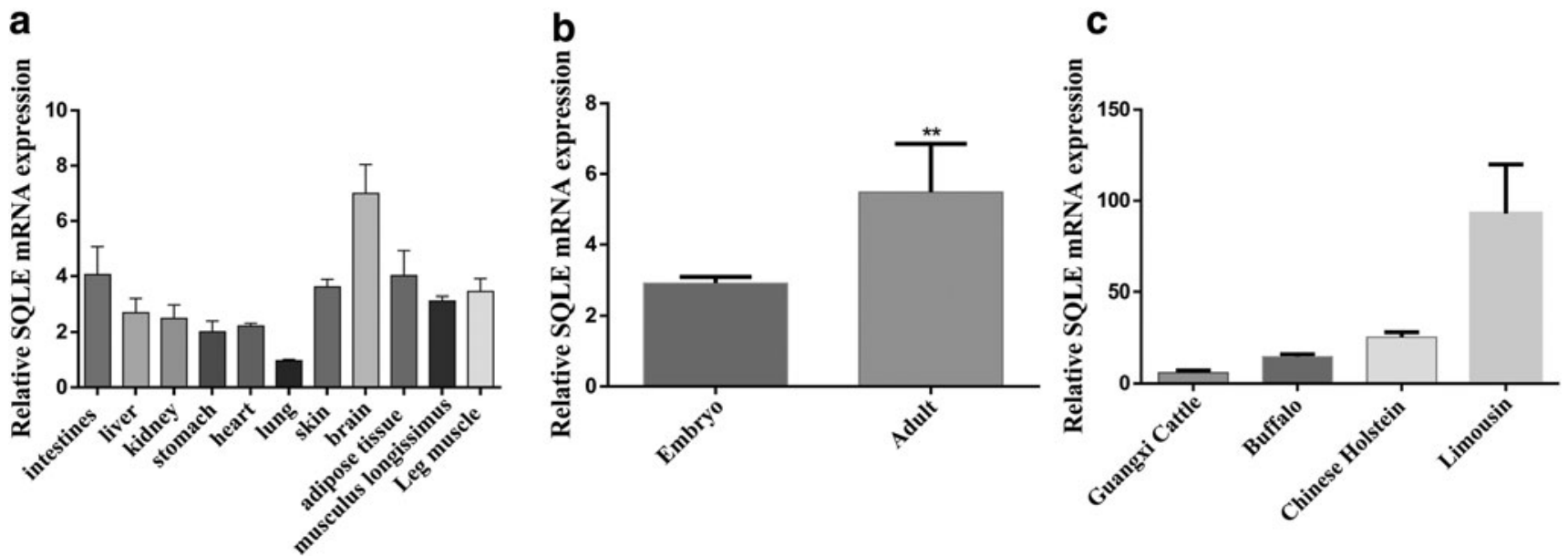

FIG. 1. The expression characteristics of bovine SQLE gene. (a) The expression of SQLE in different tissues of Guangxi cattle at embryonic stage. (b) The expression of SQLE in skeletal muscle of Guangxi cattle at the embryonic stage compared with the adult stage. (c) The expression of SQLE in skeletal muscle of four varieties of beef cattle at the adult stage. Bars labeled with asterisk mean significantly different $(p<0.05)$. SQLE, Squalene epoxidase. 
a

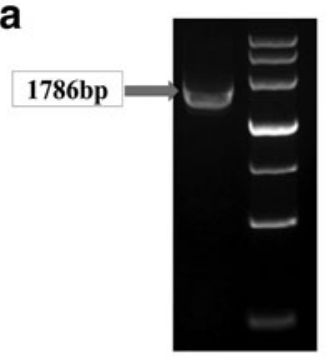

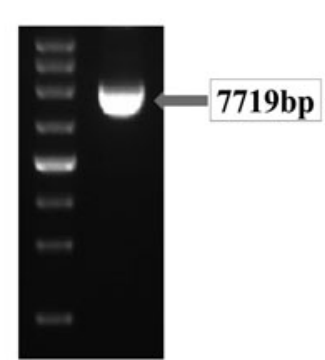
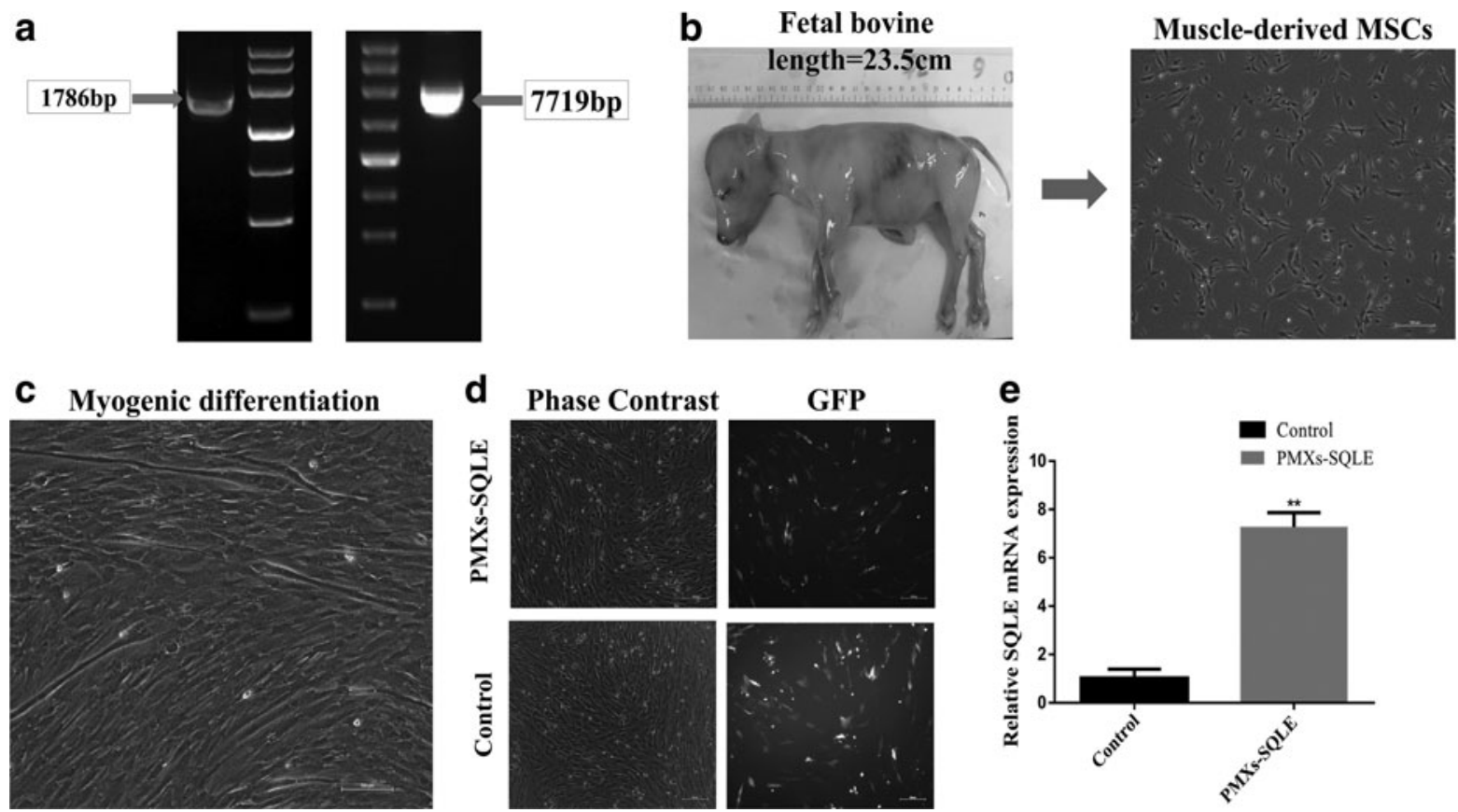

FIG. 2. The establishment of skeletal muscle-derived MSC lines that overexpress bovine SQLE. (a) Cloning of SQLE gene and construction of its expression vector. (b, c) Separation of the primary bovine skeletal muscle-derived MSCs and the myogenic induction of MSCs. (d, e) Cell transfection of SQLE overexpression vector and detection of its expression efficiency. Bars labeled with asterisk mean significantly different $(p<0.05)$ (scale bars $=100 \mu \mathrm{m})$. MSC, mesenchymal stem/stromal cell.

(Fig. 1a). Moreover, compared with the expression of the longest muscle tissue in the embryonic period, $S Q L E$ was significantly upregulated in adulthood $(P<0.05)$ (Fig. 1b). It was worth noting that among the four different beef cattle breeds, $S Q L E$ had the highest expression in the longissimus muscle tissue of Limousin, and the lowest expression in Guangxi cattle (Fig. 1c).

\section{The establishment of skeletal muscle-derived MSC lines that overexpressed bovine SQLE}

In this study, we constructed a SQLE overexpression vector by homologous recombination technology, and analyzed by sequencing and transfection of HEK293T cells (Supplementary Fig. S1), indicating that the constructed plasmid vector can be used in subsequent experiments (Fig. 2a, b). At the same time, MSCs from bovine skeletal muscle were isolated by $0.2 \%$ Roche type I collagenase. Under the microscope, we observed that these cells were spindle like, with strong refractive index and spiral growth (Fig. 2c).

These skeletal muscle-derived MSCs could be cultured for more than 10 generations during the culture. Based on the CCK-8 data, we found that skeletal muscle-derived MSCs had a large cell proliferation rate during the proliferative phase, and the shape is fusiform, and has a threedimensional effect. Moreover, these skeletal muscle-derived MSCs could differentiate into myotubes when cultured in an induction medium containing $2 \%$ horse serum (Fig. 2d).

Afterward, $P M X s-S Q L E$ plasmid was introduced into the bovine skeletal muscle-derived MSCs by a transfection reagent. After 24 hours, the reporter gene green fluorescence protein (GFP) was expressed in the transfected cells, which with strong fluorescence was observed under the fluorescence microscope (Fig. 2e). Further qPCR was carried out to detect the expression level of SQLE in the transfected cells. Compared with the control group, the results showed that the 7.09-fold SQLE expression level increased was ectopic for the transfected group (Fig. 2f).

It indicated that a skeletal muscle-derived MSCs line overexpressing bovine $S Q L E$ was successfully established. In addition, the morphological differences between the overexpressed SQLE cell line and the WT cells and control cells were minimal.

\section{SQLE inhibits proliferation of bovine skeletal muscle-derived MSCs}

According to our observations, compared with the control group, the proliferation rate of bovine skeletal musclederived MSCs changed significantly after overexpression of the SQLE gene. We initially used the CCK-8 Kits to check viability of cells, and noted significant difference in cell proliferation between control group and SQLEoverexpressing group $(p<0.05)$, showing that overexpression of $S Q L E$ inhibits MSC proliferation (Fig. 3a). These observations were also reinforced by our EdU analyses, which also showed a significant difference between the control group and $S Q L E$-overexpressing group, and $S Q L E$ overexpressing reduces the number of EdU-positive cells (Fig. 3b).

On the other hand, cell cycle analysis revealed that $S Q L E-$ overexpressing group increased the number of MSCs in $\mathrm{S}$ phases and decreased the proportion of cells in G2 phase, 
a

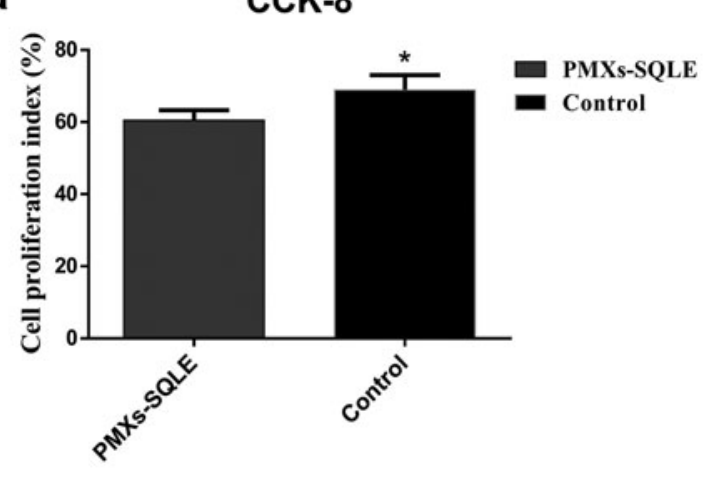

b

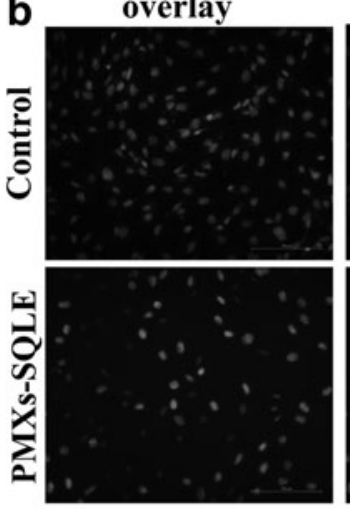

EdU

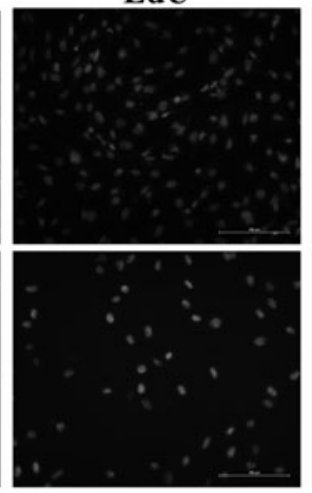

DAPI

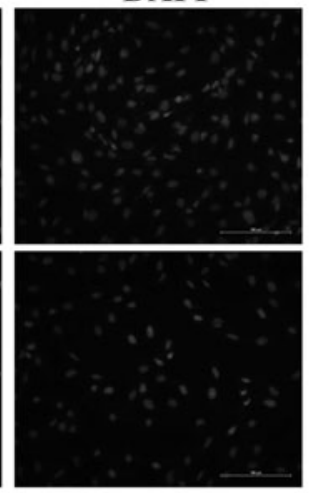

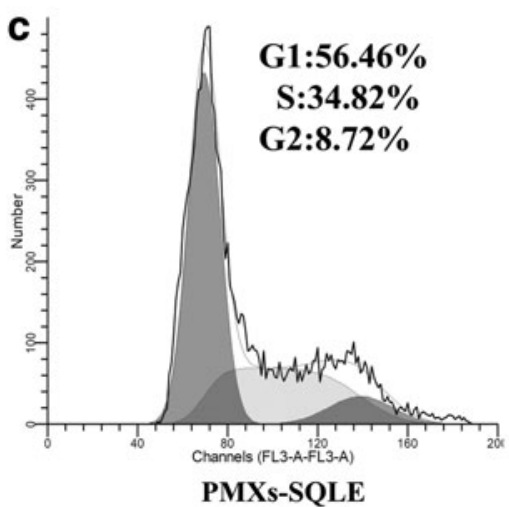
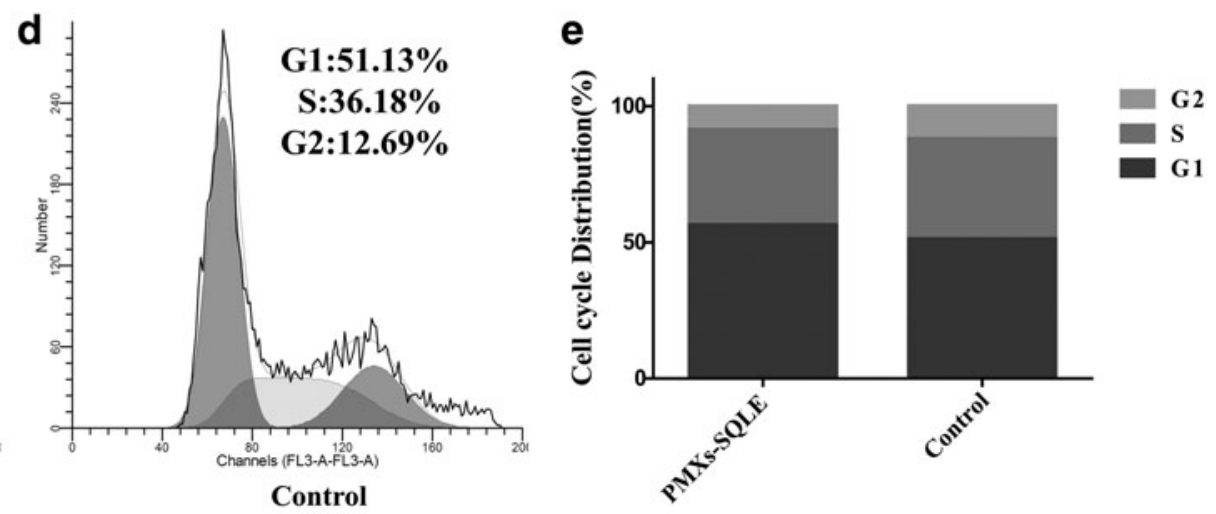

FIG. 3. SQLE affects proliferation of bovine skeletal muscle-derived MSCs. (a) Cell proliferation index was detected by CCK-8 assay. (b) Cell proliferation was detected with 5-ethynyl-20-deoxyuridine (EdU). (c-e) Bovine skeletal musclederived MSCs were transfected with SQLE overexpressing and cell phase analyzed by flow cytometry. Bars labeled with asterisk means significantly different $(p<0.05)$ (scale bars $=100 \mu \mathrm{m})$. CCK-8, Cell Counting Kit-8; EdU, 5-ethynyl-20deoxyuridine.

and had effect on the proportion of cells in the $\mathrm{S}+\mathrm{G} 2$ phases, suggesting that $S Q L E$ may have effect on cell proliferation (Fig. 3c-e). Seeking to explore these observed differences in greater detail, we conducted qPCR analysis targeting known markers of cell proliferation, including proliferating cell nuclear antigen $(P C N A)$, cyclinD1, and $B c l-2$. This analysis showed that all of these markers of cell proliferation had significantly higher levels in control group than that in $S Q L E$-overexpressing group $(p<0.05$, Fig. $4 c)$. These findings strongly suggest that the overexpression of $S Q L E$ inhibits the proliferation of bovine skeletal musclederived MSCs.

\section{SQLE promotes apoptosis of bovine skeletal muscle-derived MSCs}

The bovine skeletal muscle-derived MSCs were transfected with the control plasmid and the SQLE-overexpressing plasmid. After 24 hours of culture, the results showed that the $S Q L E$-overexpressing group had more cell suspension than the control group. To confirm the effect of the SQLE gene on bovine skeletal muscle-derived MSCs, we next performed the Annexin V-FITC/Hoechst 33342 and PI staining to check viability of MSCs (Fig. 4a, b), and noted significant difference in cell death between control group and $S Q L E$ overexpressing group $(p<0.05)$, suggesting that $S Q L E$ may have an effect on cell apoptosis.
Seeking to explore these observed differences in greater detail, we conducted qPCR analysis targeting known markers of cell apoptosis, including Bax, Caspase9, p21, and $p 53$. This analysis showed that all of these markers of cell apoptosis had significantly higher levels in SQLEoverexpressing group than in the control group $(p<0.05$, Fig. 4c). These findings strongly suggest that the overexpression of $S Q L E$ promotes the apoptosis of bovine skeletal muscle-derived MSCs.

\section{SQLE promotes differentiation of bovine skeletal muscle-derived MSCs}

The effect of the SQLE gene on myogenic differentiation of bovine skeletal muscle-derived MSCs was investigated. First, the mRNA expression of the SQLE and the markers $M y o D 1$, myogenin $(M y o G)$, and myosin heavy chains $(\mathrm{MyHC})$ were detected at 0 and 4 days after MSC myogenesis (Fig. 5a). The results showed that these genes were significantly upregulated on the fourth day. Next, we transfected the SQLE overexpression vector on the cells and continued to culture for 24 hours, followed by replacement of the medium with myogenic induction medium. On the fourth day, we used the immunofluorescence assay to analyze the differentiation of bovine skeletal muscle-derived MSCs, and noted myotube differences in cell differentiation between control group and $S Q L E$-overexpressing group 

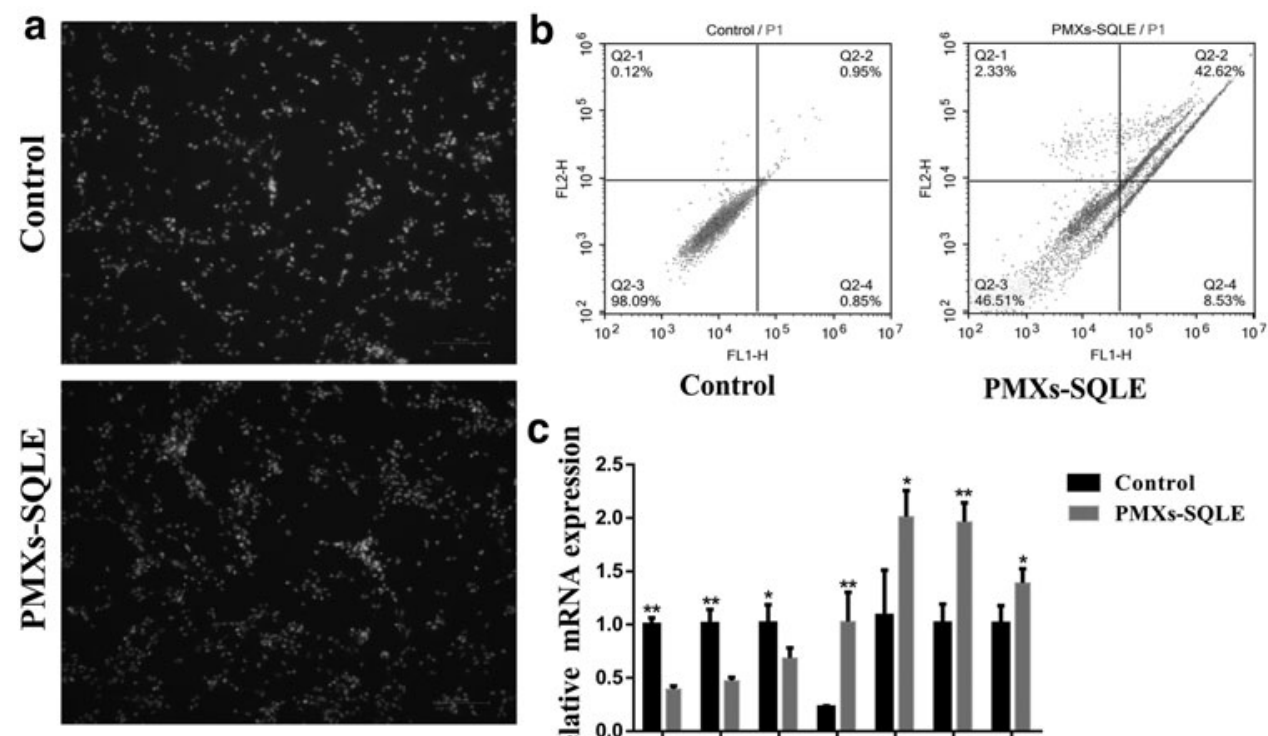

C

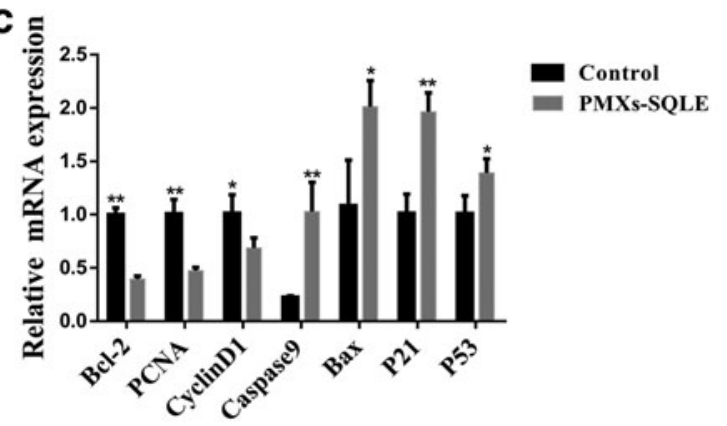

FIG. 4. SQLE promotes apoptosis of bovine skeletal muscle-derived MSCs. Bovine skeletal muscle-derived MSCs were transfected with SQLE, and cell apoptosis was determined (a) and counted (b) by Annexin $\mathrm{V}$-FITC/PI binding followed by flow cytometry. (c) The mRNA expression of proliferation and apoptosis marker genes were detected by realtime qPCR. Bars labeled with asterisk mean significantly different $(p<0.05)$ (scale bars $=100 \mu \mathrm{m}$ ). qPCR, quantitative polymerase chain reaction. V-FITC/PI, V-Fluorescein

Isothiocyanate/Propidium Iodide.
(Fig. 5b), suggesting that $S Q L E$ overexpressing promoted $M y o D 1$ expression and formation of myotube.

At the same time, seeking to explore these observed differences in greater detail, we conducted qPCR analysis targeting known markers of myoblast differentiation, including $S Q L E, M y o D 1, M y o G$, and $M y H C$. This analysis showed that all of these markers of myoblast differentiation had significantly higher levels in $S Q L E$-overexpressing group than that in control group ( $p<0.05$, Fig. 5c). These findings strongly suggest that the overexpression of $S Q L E$ improves the ability of differentiation.

\section{Discussion}

Although our results are preliminary, the effect that we observed from overexpression of SQLE on differentiation of muscle cells indicates that this type of bio-technological manipulation may offer an attractive approach to breeders and other technologists seeking to improve meat production from cattle (Guo et al., 2017). Our results demonstrate several clear influences for $S Q L E$ relating to differentiation and proliferation in bovine skeletal muscle-derived MSCs.
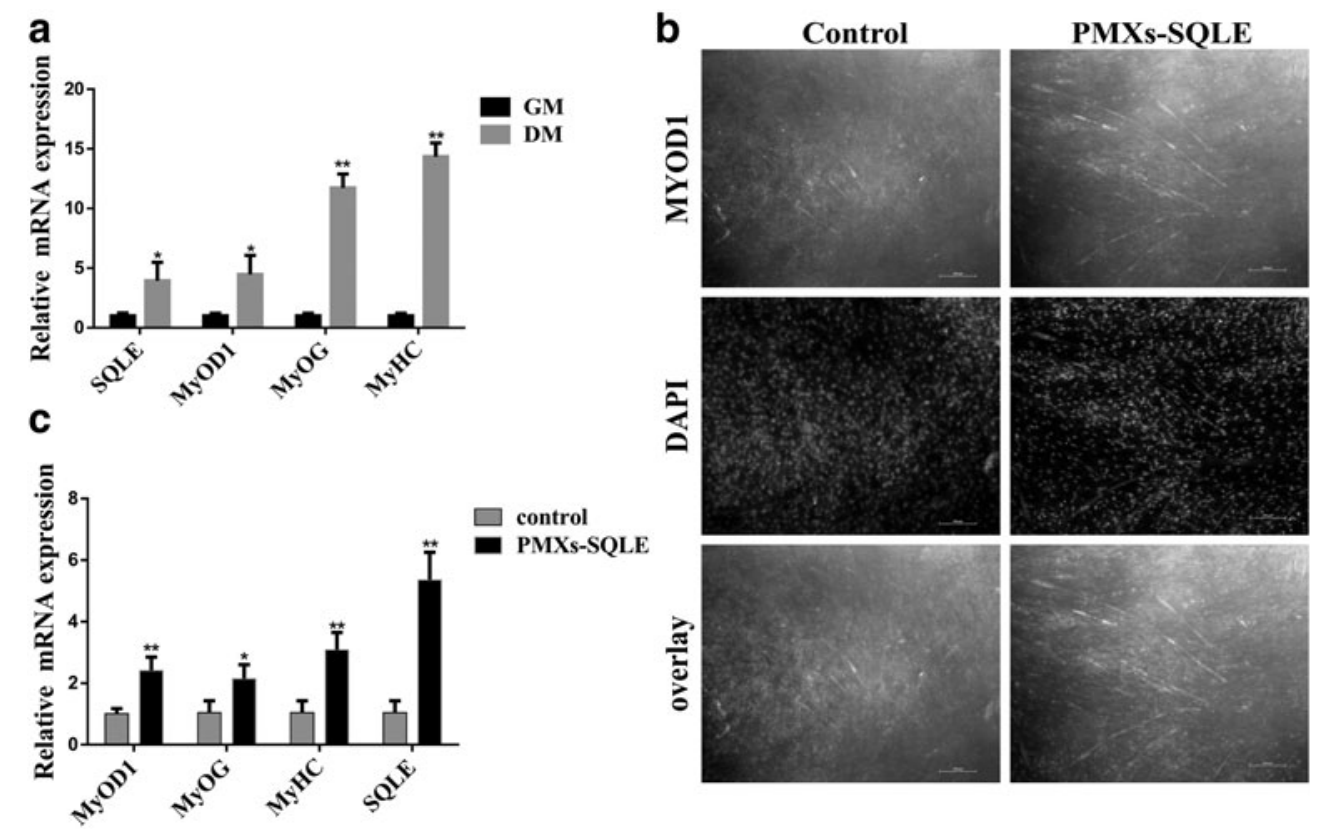

FIG. 5. SQLE promotes differentiation of bovine skeletal muscle-derived MSCs. (a) The mRNA expression of SQLE and differentiation marker genes were detected by qPCR. (b, c) Bovine skeletal muscle-derived MSCs were transfected with SQLE, and cell differentiation was detected by immunofluorescence (MyoD1) and by real-time PCR (SQLE, MyoD1, MyoG, MyHC). Bars labeled with asterisk mean significantly different $(p<0.05)$ (scale bars $=100 \mu \mathrm{m})$. MyHC, myosin heavy chains. 
We found in bovine skeletal muscle-derived MSCs that $S Q L E$ clearly affects differentiation and proliferation. However, it remains to be seen whether or not similar effects occur in whole animals, and it is also unknown if such effects of $S Q L E$ will ultimately control improvements in meat production. Reviewing the results of scientists, knocking down the expression of the $S Q L E$ gene in pigs, inhibits adipogenesis and myogenesis, whereas significantly reduced the level of cellular reactive oxygen species (ROS). On the other hand, $S Q L E$ was one of the significantly differentially expressed genes in different marbled muscle tissues of cattle.

The foregoing studies suggest that the bovine SQLE gene not only affects myogenesis, but may also affect intramuscular fat production and intracellular ROS levels (Ha et al., 2017). Therefore, the role of SQLE in the regulation of adipogenesis and intracellular ROS levels in bovine skeletal muscle MSCs will be one of our next research contents. As in the lncSEMT (a lncRNA) study, scientists have successfully produced genetically engineered sheep using embryo transfer technology, and their growth performance has been significantly improved (Wei et al., 2018). Of course, we can also follow their research methods to verify the impact of $S Q L E$ on animal performance and should clarify these topics.

Although overexpression of SQLE gene in bovine skeletal muscle MSCs inhibits proliferation and promotes differentiation, its mechanism is still unknown, and whether SQLE can be a myogenic differentiation marker like MyoDl and $M y o G$, and whether it participates in cell energy metabolism, etc. is worth exploring also to verify more information.

Nowadays, the development of biomultiomics is very rapid, which provides us with advantageous methods and technical means for further exploring the mechanism of DNA methylation, histone modification (Bodega et al., 2017; Creyghton et al., 2010; Yan et al., 2016), lncRNAs (Butchart et al., 2016; Guo et al., 2017; Jin et al., 2017), and circRNAs expression in the regulation of muscle formation of bovine skeletal muscle MSCs by SQLE (Cherubini et al., 2019; Legnini et al., 2017). Then, we will use our previous research results, combining with CRISPR/Cas9 technology to produce $S Q L E$ gene-edited animals (Hryhorowicz et al., 2017; J and Das, 2019), and verify the regulatory role of $S Q L E$ on animal production performance. Therefore, we need to carry out follow-up studies to analyze the role of $S Q L E$ in regulating the development of bovine skeletal muscle-derived MSCs.

\section{Author Disclosure Statement}

The authors declare they have no conflicting financial interests.

\section{Funding Information}

This work was supported by the National Natural Science Foundation of China (No. 31860644, 31760334), Science and Technology Bureau Project of Guangxi Nanning (No.20194147), Opening of Key Laboratory of Livestock Genetic Improvement in Guangxi (2018GXKLLGI-04), and Science and Technology Major Project of Guangxi (AA17204051).

\section{Supplementary Material}

Supplementary Figure S1

Supplementary Table S1

\section{References}

Beane, O.S., Fonseca, V.C., Cooper, L.L., Koren, G., and Darling, E.M. (2014). Impact of aging on the regenerative properties of bone marrow-, muscle-, and adiposederived mesenchymal stem/stromal cells. PLoS One. 9, e115963.

Bodega, B., Marasca, F., Ranzani, V., Cherubini, A., Della Valle, F., Neguembor, M.V., Wassef, M., Zippo, A., Lanzuolo, C., Pagani, M., and Orlando, V. (2017). A cytosolic Ezh1 isoform modulates a PRC2-Ezh1 epigenetic adaptive response in postmitotic cells. Nat. Struct. Mol. Biol. 24, 444-452.

Butchart, L.C., Fox, A., Shavlakadze, T., and Grounds, M.D. (2016). The long and short of non-coding RNAs during postnatal growth and differentiation of skeletal muscles: Focus on lncRNA and miRNAs. Differentiation. 92, 237-248.

Camernik, K., and Zupan, J. (2019). Complete assessment of multilineage differentiation potential of human skeletal muscle-derived mesenchymal stem/stromal vells. Methods Mol. Biol. 2045:131-144.

Cherubini, A., Barilani, M., Rossi, R.L., Jalal, M.M.K., Rusconi, F., Buono, G., Ragni, E., Cantarella, G., Simpson, H., Peault, B., and Lazzari, L. (2019). FOXP1 circular RNA sustains mesenchymal stem cell identity via microRNA inhibition. Nucleic. Acids Res. 47, 5325-5340.

Creyghton, M.P., Cheng, A.W., Welstead, G.G., Kooistra, T., Carey, B.W., Steine, E.J., Hanna, J., Lodato, M.A., Frampton, G.M., Sharp, P.A., Boyer, L.A., Young, R.A., and Jaenisch, R. (2010). Histone H3K27ac separates active from poised enhancers and predicts developmental state. Proc. Natl. Acad. Sci. U. S. A. 107, 21931-21936.

Du, M., and Carlin, K.M. (2012). Meat science and muscle biology symposium: Extracellular matrix in skeletal muscle development and meat quality. J. Anim. Sci. 90, 922923.

Duarte, M.S., Gionbelli, M.P., Paulino, P.V., Serao, N.V., Silva, L.H., Mezzomo, R., Dodson, M.V., Du, M., Busboom, J.R., Guimaraes, S.E., and Filho, S.E. (2013). Effects of pregnancy and feeding level on carcass and meat quality traits of Nellore cows. Meat. Sci. 94, 139-44.

Garcia-Bermudez, J., Baudrier, L., Bayraktar, E.C., Shen, Y., La, K., Guarecuco, R., Yucel, B., Fiore, D., Tavora, B., Freinkman, E., Chan, S.H., Lewis, C., Min, W., Lnghirami, G., Sabatini, D.M., and Birsoy, K. (2019). Squalene accumulation in cholesterol auxotrophic lymphomas prevents oxidative cell death. Nature. 567, 118-122.

Guo, Y., Wang, J., Zhu, M., Zeng, R., Xu, Z., Li, G., and Zuo, B. (2017). Identification of MyoD-responsive transcripts reveals a novel long non-coding RNA (IncRNA-AK143003) that negatively regulates myoblast differentiation. Sci. Rep. 7, 2828.

Ha, J., Kwon, S., Hwang, J.H., Park, D.H., Kim, T.W., Kang, D.G., Yu, G.E., Park, H.C., An, S.M., and Kim, C.W. (2017). Squalene epoxidase plays a critical role in determining pig meat quality by regulating adipogenesis, myogenesis, and ROS scavengers. Sci. Rep. 7, 16740.

Hernandez-Hernandez, J.M., Garcia-Gonzalez, E.G., Brun, C.E., and Rudnicki, M.A. (2017). The myogenic regulatory factors, determinants of muscle development, cell identity and regeneration. Semin. Cell Dev. Biol. 72, 10-18. 
Hryhorowicz, M., Lipinski, D., Zeyland, J., and Slomski, R. (2017). CRISPR/Cas9 immune system as a tool for genome engineering. Arch. Immunol. Ther. Exp. 65, 233-240.

J, B., and Das, A. (2019). An edible fungi Pleurotus ostreatus inhibits adipogenesis via suppressing expression of PPAR gamma and C/EBP alpha in 3T3-L1 cells: In vitro validation of gene knock out of RNAs in PPAR gamma using CRISPR spcas9. Biomed. Pharmacother. 116, 109030.

Jin, C.F., Li, Y., Ding, X.B., Li, X., Zhang, L.L., Liu, X.F., and Guo, H. (2017). lnc133b, a novel, long non-coding RNA, regulates bovine skeletal muscle satellite cell proliferation and differentiation by mediating miR-133b. Gene. 630, 35-43.

Legnini, I., Di Timoteo, G., Rossi, F., Morlando, M., Briganti, F., Sthandier, O., Fatica, A., Santini, T., Andronache, A., Wade, M., Laneve, P., Rajewsky, N., and Bozzoni, I. (2017). Circ-ZNF609 is a circular RNA that can be translated and functions in myogenesis. Mol. Cell 66, 22-37. e9.

Li, H., Wei, X., Yang, J., Dong, D., Hao, D., Huang, Y., Lan, X., Plath, M., Lei, C., Ma, Y. Lin, F. Bai, Y., and Chen, H. (2018a). circFGFR4 promotes differentiation of myoblasts via binding miR-107 to relieve its inhibition of Wnt3a. Mol. Ther. Nucleic Acids. 11, 272-283.

Li, H., Yang, J., Jiang, R., Wei, X., Song, C., Huang, Y., Lan, X., Lei, C., Ma, Y., Hu, L., and Chen, H. (2018b). Long noncoding RNA profiling reveals an abundant MDNCR that promotes differentiation of myoblasts by sponging miR-133a. Mol. Ther. Nucleic Acids 12, 610-625.

Li, Y., Chen, X., Sun, H., and Wang, H. (2018c). Long noncoding RNAs in the regulation of skeletal myogenesis and muscle diseases. Cancer Lett. 417, 58-64.

Mok, G.F., Lozano-Velasco, E., and Munsterberg, A. (2017). microRNAs in skeletal muscle development. Semin. Cell Dev. Biol. 72, 67-76.

Nakamura, Y., Miyaki, S., Ishitobi, H., Matsuyama, S., Nakasa, T., Kamei, N., Akimoto, T., Higashi, Y., and Ochi, M. (2015). Mesenchymal-stem-cell-derived exosomes accelerate skeletal muscle regeneration. FEBS Lett. 589, 1257-1265.

Wei, C., Wu, M., Wang, C., Liu, R., Zhao, H., Yang, L., Liu, J., Wang, Y., Zhang, S., Yuan, Z. Liu, Z. Hu, S. Chu, M. Wang, $\mathrm{X}$., and Du, L. (2018). Long noncoding RNA lnc-SEMT modulates IGF2 expression by sponging miR-125b to pro- mote sheep muscle development and growth. Cell. Physiol. Biochem. 49, 447-462.

Wei, X., Li, H., Yang, J., Hao, D., Dong, D., Huang, Y., Lan, X., Plath, M., Lei, C., Lin, F. Bai, Y., and Chen, H. (2017). Circular RNA profiling reveals an abundant circLMO7 that regulates myoblasts differentiation and survival by sponging miR-378a-3p. Cell Death Dis. 8, e3153.

Yan, L., Guo, H., Hu, B., Li, R., Yong, J., Zhao, Y., Zhi, X., Fan, X., Guo, F., Wang, X. Wang, W. Wei, Y. Wang, Y. Wen, L. Qiao, J., and Tang, F. (2016). Epigenomic landscape of human fetal brain, heart, and liver. J. Biol. Chem. 291, 4386-98.

Zammit, P.S. (2017). Function of the myogenic regulatory factors Myf5, MyoD, Myogenin and MRF4 in skeletal muscle, satellite cells and regenerative myogenesis. Semin. Cell Dev. Biol. 72, 19-32.

Zhu, P., Li, H.Y., Huang, G.T., Cui, J.Y., Zhang, R.M., Cui, K.Q., Yang, S.F., and Shi, D.S. (2018). Molecular cloning, identification, and expression patterns of myostatin gene in water buffalo (Bubalus Bubalis). Anim. Biotechnol. 29, 26-33.

Address correspondence to: Sufang Yang

State Key Laboratory for Conservation and Utilization of Subtropical Agro-Bioresources College of Animal Science and Technology

Guangxi University Nanning 530005

Guangxi, China

E-mail: ysfang@gxu.edu.cn

Bingkun Xie Guangxi Key Laboratory of Livestock Genetic Improvement Guangxi Institute of Animal Sciences Nanning 530001 Guangxi, China

E-mail: bkxie@163.com 